Reprintẹd from

\author{
The \\ BRITISH JOURNAL \\ OF RADIOLOGY
}

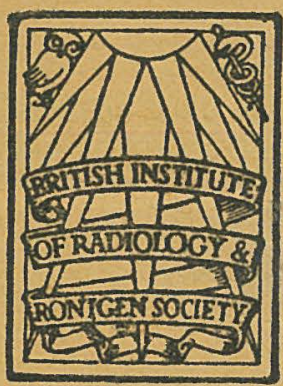

REF

MEL 


\title{
UNDULANT FEVER SPONDYLITIS
}

\author{
By F. Zammit, M.D., D.M.R. \\ St. Luke's Hospital, Malta
}

(Received February, 1958)

$T^{\mathrm{T}}$ HERE are very few reports in the radiological literature of bone involvement of the spine by the Brucella Melitensis.

The objective findings in the first proved case (Kulowski and Vinke, 1932), were involvement of the intervertebral facets and a psoas abscess.

All the cases diagnosed as Brucellar spondylitis by the orthopaedic department during 1947-54 were reviewed. During this period 6301 cases of undulant fever were reported (Report on Medical and Health Department, Malta, 1954), in 115 of which spondylitis was diagnosed. I am including in this paper 62 cases in which there were definite radiological signs of involvement of the spine, but the intervertebral facets were rarely affected. be acute or insidious. The course of undulant fever is very irregular and the symptoms are exceedingly variable in nature and severity.

The main symptom of this disease is a highly irregular fever, rising and falling, forming a series of irregular waves, which goes on for long periods and is often associated with headaches, enlargement of the spleen, excessive perspiration, secondary anaemia, weakness and, towards the end of the disease, pains of a rheumatic type, which may be very severe.

The disease varies enormously in its duration from weeks to several months and at times even to years, but its course is frequently interrupted by various complications, the most common being,

TABLE I

Incidence of undulant fever in Malta (annual reports on Medical and Health Department, Malta) 1944-1954

\begin{tabular}{|c|c|c|c|c|c|c|c|c|c|c|}
\hline 1944 & 1945 & 1946 & 1947 & 1948 & 1949 & 1950 & 1951 & 1952 & 1953 & 1954 \\
\hline 173 & 1024 & 2410 & 1390 & 1039 & 902 & 834 & 613 & 550 & 425 & 548 \\
\hline
\end{tabular}

\section{Historical notes}

Brucella melitensis, the causative organism of undulant fever, was first isolated by Bruce (1887). Wright and Semple (1897) demonstrated that the organism discovered by Bruce was agglutinated by the serum of those affected by this disease. The host of Brucella melitensis is the goat. This fact was discovered by Zammit (1905). Goats, the chief source of the milk supply, are frequently infected. According to a survey which is being carried out by the Health Department since 1955, the serum agglutination reaction has been found positive in 520 out of 1544 goats examined. The incidence of the disease in Malta has been greatly reduced since the introduction under Government control of a system of pasturising the milk supply (Table I).

\section{Clinical features}

In Malta undulant fever is endemic and causes a lot of disability. It is commonest in children under ten years (Table II). There is little difference in sex incidence. The disease is relatively mild in children and old people. The incubation period varies, usually being about 14 days. The onset may bronchitis, bronchopneumonia, hepatitis, perisplenitis and orchitis. The patient becomes progressively emaciated and anaemic, sometimes to a cachectic degree. Eventually the fever disappears, but convalescence drags on for a considerable time and is usually disturbed by weakness and severe pains of a rheumatic nature in one or more regions where there are no radiological signs of bone or joint involvement, or by localised persistent pain from bone involvement. The most common sites of these pains are the neck, shoulder, Iumbar region, hip, thigh and knees. Complete resolution is the rule and there are no after effects, except occasional stiffness. The rheumatic phase may last from 15 days to three months, the average being four weeks. Abortion does not occur in ordinary cases; in severe cases with high temperature abortion is common.

\section{Pathology}

Undulant fever is a septicaemic infection of variable severity and duration. The changes found on post-mortem examination are not characteristic and the diagnosis is usually made by culturing the organism from the spleen, liver or other organs. 


\section{F. Zammit}

Eyre (1908) first showed at necropsy that the bone marrow may be involved in Brucellosis and obtained the organism from that tissue.

Burnett (1922) found that it was cultivable from the bone marrow in some cases when it was not cultivable from the blood.

Epitheloid nodules in the human bone marrow were first described by Wohlwill (1932). These nodules are of the infective granuloma type and resemble the tubercle.

TABLE II

AGE AND SEX DISTRIBUTION, 1954 (ANNUAL REPORT--MEDICAL and Health Department, Malta, 1954)

\begin{tabular}{|c|c|c|c|}
\hline Age & & Males & Females \\
\hline Under 4 years & $\cdots \quad \ldots$ & 23 & 20 \\
\hline 4 years $\quad \ldots$ & $\cdots \quad \ldots$ & 14 & 14 \\
\hline 5 to 9 years & $\cdots \quad \ldots$ & 57 & 62 \\
\hline 10 to 14 years & $\ldots$ & 33 & 25 \\
\hline 15 to 19 years & $\ldots \quad \ldots$ & 33 & 24 \\
\hline 20 to 24 years & $\ldots \quad \ldots$ & 24 & 18 \\
\hline 25 to 34 years & . & 38 & 23 \\
\hline 35 to 44 years & . & 20 & 25 \\
\hline 45 years and over. & $\ldots$ & 48 & 48 \\
\hline Total & $\begin{array}{ll}\ldots & \ldots\end{array}$ & 290 & 258 \\
\hline
\end{tabular}

Sundberg and Spink (1947) have described these granulomatous nodules as varying in size and consisting primarily of epitheloid and giant cells, surrounded by many lymphocytes and other mononuclear cells. Reticulo-endothelial cells with irregular cell outline were also prominent. Langhans type of giant cell was also noted. Focal necrosis has been reported as occurring in human lesions (Löffler and Albertini, 1930; Meyer, 1943; Rabson, 1939; Wegener, 1935; Wohlwill, 1932), but when this occurs it is only partial. It is difficult to differentiate miliary tuberculosis, sarcoidosis and Brucellosis as they occur in the marrow. Hamilton (1954) described the granuloma of Brucellosis as being smaller and less distinctly limited than in sarcoidosis and miliary tuberculosis.

\section{Diagnosis}

Diagnosis is difficult in the early stages. The best way to avoid missing Brucellosis in cases which present themselves with vague symptoms and irregular fever is to test the blood for the aggluti- nation reaction. This is particularly indicated in mild cases with an insidious onset.

A serum agglutination reaction of $1: 80$ is regarded as diagnostic. In some cases the reaction does not rise above $1: 40$. A rising titre is indicative of active Brucellosis. The serum agglutination test may be negative in the chronic phase or in the very acute stage. In several cases a positive agglutination test was obtained in synovial fluid from affected joints and in pus aspirated from abscesses in the vicinity of diseased bones. The organism has also been cultivated from these sources in several cases. Blood culture is not always positive. Bone marrow examination may help in the diagnosis of the difficult cases, especially when it is drawn from the sites which show radiological signs of bone involvement.

\section{Blood changes}

In the early and in the acute stages of the disease a leucopaenia is commonly present. A low mononuclear count is present in practically all cases in the acute stage (Rainsford, 1935). Lymphocytosis is generally present (Sharp, 1934). In a few cases with high white counts polymorphs predominate, which is probably due to the presence of a superadded secondary infection.

Blood sedimentation rate was, as a general rule, high in the cases which showed radiographic signs of involvement of the spine.

\section{Signs and symptoms}

\section{Spondylitis}

The signs and symptoms of spondylitis manifest themselves during the period of convalescence in the typical case. In the chronic atypical cases it is difficult to ascertain the pre-existence of undulant fever, because the prodromal symptoms are vague and as a general rule have been present for longer periods.

The earliest and constant symptom is pain, localised to the affected region of the spine. It is usually progressive and can be severe. It is made worse by movements and is relieved by rest. Referred pains to the limbs and in some cases the girdle type occurred in 60 per cent of this series.

Restricted movements and spasm of the spinal muscles were commonly present.

Localised tenderness was present in 20 per cent of these cases.

Signs of cord compression with paraplegia or less marked signs of motor or sensory disturbances were found in eight cases. In another case there were no signs of bone changes, but myelography showed a block at the level of the third lumbar vertebra and 


\section{Undulant Fever Spondylitis}

at operation a peridural abscess was found at this site. This case has not been included in this series.

\section{Radiographic changes}

In the early stages of the disease, even though localised pain is very marked and the clinical signs of spondylitis are present, the radiograph may not show any abnormal changes in the vertebrae. This
In most cases more than one vertebra was involved, usually two, but there were cases in which three or four vertebrae were affected. In some cases bone changes at different levels were present with sound vertebrae in betwen.

The radiological changes that occur in the spine in undulant fever can be described under four headings:

TABLE III

AGE INCIDENCE OF SPONDYLITIS

\begin{tabular}{|c|c|c|c|c|c|c|}
\hline $\begin{array}{c}\text { Under 10 } \\
\text { years }\end{array}$ & $\begin{array}{c}10-20 \\
\text { years }\end{array}$ & $\begin{array}{c}21-30 \\
\text { years }\end{array}$ & $\begin{array}{c}31-40 \\
\text { years }\end{array}$ & $\begin{array}{c}41-50 \\
\text { years }\end{array}$ & $\begin{array}{c}51-60 \\
\text { years }\end{array}$ & $\begin{array}{c}61-70 \\
\text { years }\end{array}$ \\
\hline 1 & 5 & 2 & 9 & 19 & 17 & 9 \\
\hline
\end{tabular}

latent period was found to vary in this series of cases between four weeks and four to six months, the average being three months.

Another peculiar aspect is the age of incidence (Table III). Only one third of the cases were females.

The regional distribution was as follows: cervical 5; dorsal 16; combined dorsal and lumbar 4; lumbar 37. The fourth lumbar vertebra is most commonly involved. (a) Lesions of the vertebral bodies.

(b) Changes in the disc space.

(c) Proliferative marginal reactions.

(d) Changes in the intervertebral joints.

\section{(a) Lesions of the vertebral bodies}

These can be described as (i) marginal and (ii) central.

(i) Marginal lesions. In most cases an early lesion or one of a mild degree begins at the anterior and

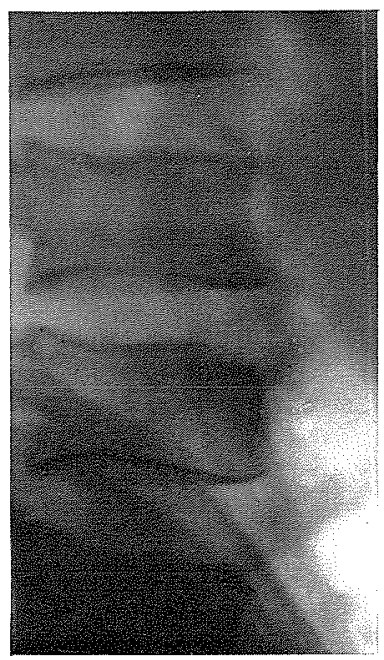

FIG. 1.

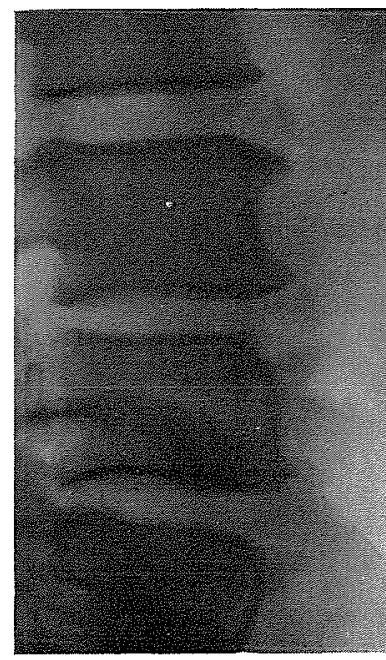

FIG. 2.

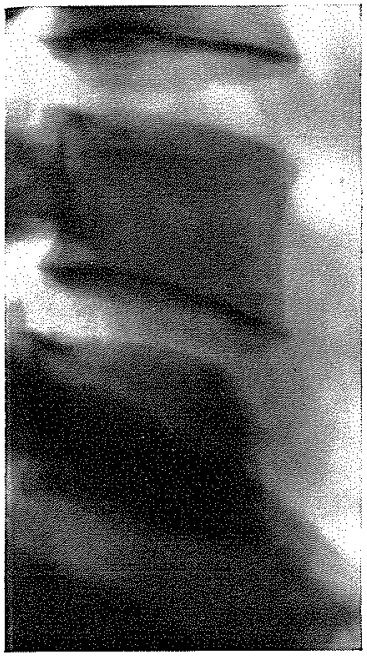

FIG. 3.

FIG. 1. C.F., 48 years. Reacts to Bruc. melit. up to $1 / 1280$. Step-like erosion upper anterior margin of L. 4 with sclerotic borders. Hazy anterior osteophytic bridging. Slight diminution of disc space. Wide disc spaces above and below affected disc.

Fig. 2. F.E., 50 years. Reacts to Bruc. melit. up to $1 / 320$. Bevel-type eroded area at supero-anterior angle of L.4, with faint intervertebral bony reaction of the central islet type, which later formed a whole bridge between the vertebrae. A similar islet is present in front of disc space above. Narrowing of disc space L.3 to L.4.

FIG. 3. M.E., 28 years. Reacts to Bruc. melit. Erosion of upper anterior angle of L. 4 with rounding off of this part of the vertebral body. Denser upper part of body. Narrowing of disc space. No intervertebral bony reaction is visible. Wide lumbo-sacral disc space. 


\section{F. Zammit}

upper margin of the body of the vertebra. This is a very characteristic feature.

It usually begins as a small ill-defined erosion or a small area of rarefaction with loss of the cortical dense line. This takes the form of a step-like deformity, an oblique cut at the angle with the triangular tip missing or simply rounding off the corner of the vertebral body (Figs. 1, 2 and 3). be described later. General rarefaction of the body involved, unlike that in tuberculosis, has not been observed. Osteoporosis, if present, is always limited and localised.

If the lesion is going to be more severe, further changes occur within the body of the vertebra. In some cases, although the process extends beyond the marginal lesion, bone destruction is not marked

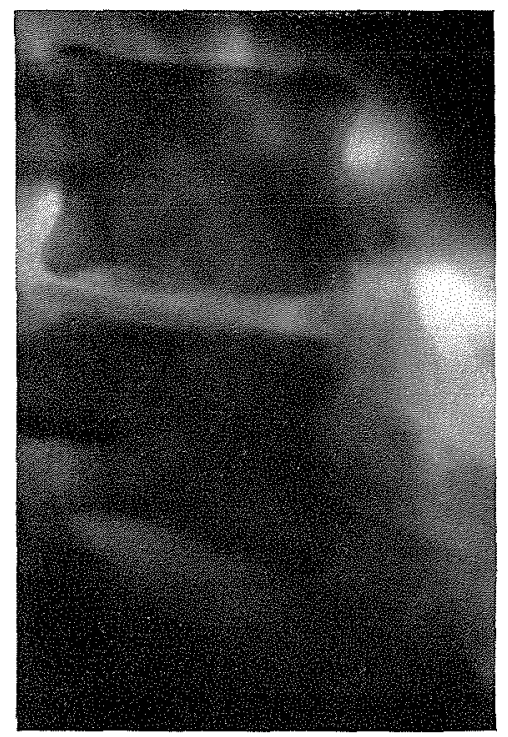

FIG. 4.

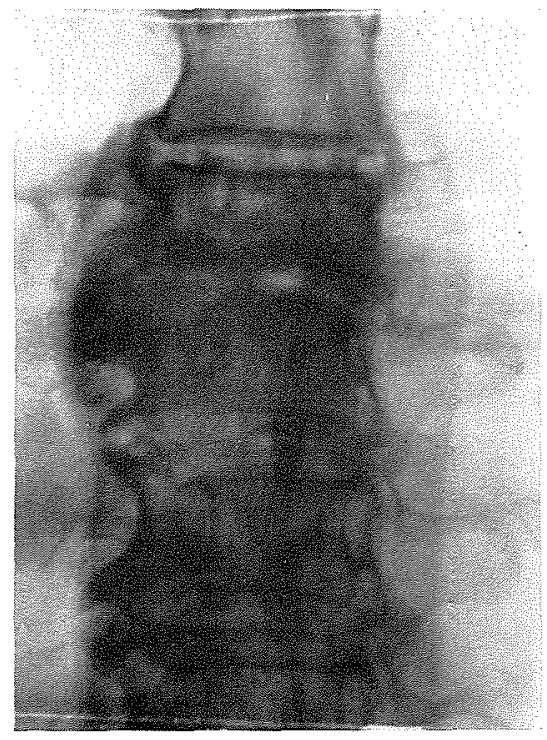

FIG. 5.

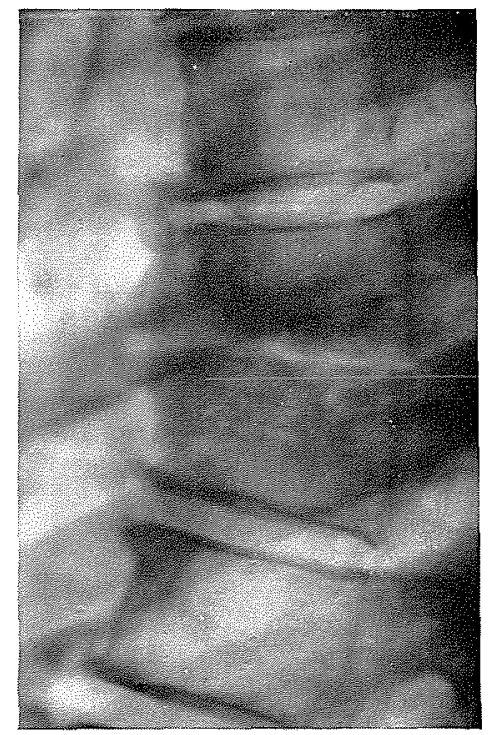

FIG. 6.

FIg. 4. A.G., 18 years. Agglutination reaction positive to Bruc. melit. Large central area of destruction of inferior border of L.4, and a smaller one at upper border of L.5, with a break at central area of surface adjoining the narrowed disc space. Irregular sclerotic patches. Erosion of anterior borders of L.4 and L.5, moth-eaten type; with slight hypertrophic bone reaction.

FIG. 5. Same case as Fig. 6. Partial collapse of D.7, with moderate intervertebral bridging at D.5-6-7; obliteration of disc space and paravertebral abscess.

FIG. 6. C.J., 60 years. Reacts to Bruc. melit. up to 1/320. Signs of cord compression. Incontinence of sphincters. Irregular erosions of disc surface of D.6-D.7, with wedging of D.6 and obliteration of disc space. Anterior intervertebral bridging. Irregular sclerotic reaction around destroyed areas, producing a coarse structure of vertebral bodies.

In a few cases a small piece of bone has been observed isolated in front of this defect.

The inner margin of such lesions is at first rarefied and ill-defined. However, in a few weeks it will change according to whether the lesion is going to be a mild or an extensive one.

If the lesion is of a mild degree, the inner area of rarefaction will eventually change into a sclerotic zone, outlining the defect. If the activity of such a process is spent, the destroyed area will eventually remould itself with irregular dense fine trabeculae and the angle of the vertebra may remain rounded and sclerosed or is turned into a projecting spur by the additional formation of a localised proliferative bone reaction (Fig. 2).

The features of these hypertrophic processes will radiologically, but a big triangular area in the vicinity of the involved angle of the vertebral body becomes cloudy, that is, it appears slightly denser and the fine trabecular structure becomes irregular and ill-defined or transformed into a homogeneous ground-glass appearance (Fig. 1). In other cases, where bone destruction is more extensive, the marginal lesion will extend towards the centre of the vertebra and along the subchondral border. When this occurs the anterior edge of the next vertebra usually shows beginning erosion as well.

The healing phase of these types of lesions is marked by encroachment of the dense zone on the translucent areas which usually disappear completely; in some cases, however, they may remain as hollow smaller areas surrounded by thick dense borders 


\section{Undulant Fever Spondylitis}

(Figs. 5 and 6 ) or rarefied areas surrounded by irregular thick trabeculae (Fig. 10).

(ii) Central lesions. Rarely, foci of destruction appear in the spongy part of the vertebral body, in the vicinity of the disc space, without any evidence of a marginal lesion. Their evolution is similar to that of the subchondral lesions above described. trabecular structures highly irregular (Figs. 8 and 9).

Some collapse of the surface near the lesions occurs, and varying degrees of wedging of the vertebral body result. This wedging or part of it may remain permanently present (Figs. 5 and 6).

These extensive lesions next to the disc surface are at times also present on the opposing surface of

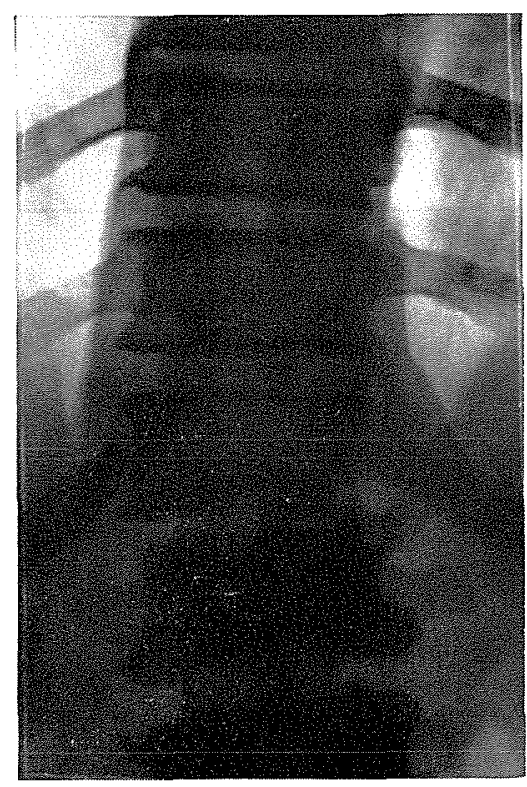

FIG. 7.

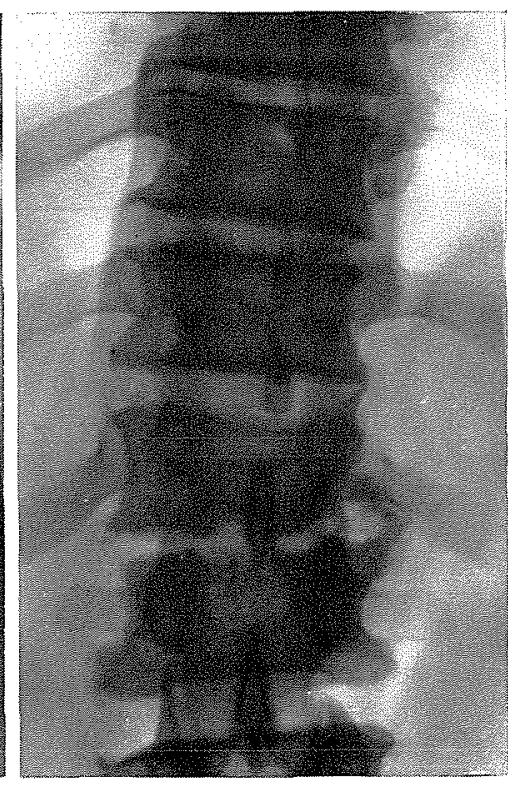

FIG. 8 .

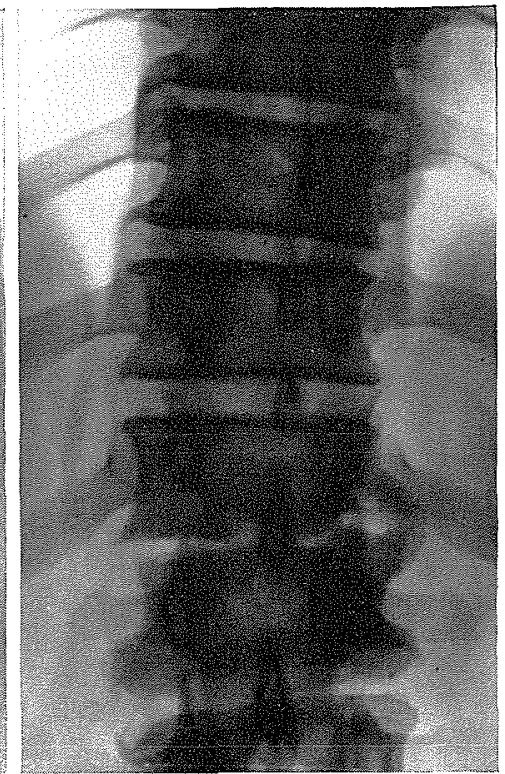

FIG. 9.

FIG. 7. C.P., 45 years. Signs of cord compression. Reacts to Bruc. melit. up to $1 / 160$. Brucella melitensis was cultivated from the pus aspirated from the paravertebral abscess. Destruction of left lower border and of central portion of lower surface of D.12. Narrowing of disc space. Paravertebral abscess shadow on the left of D.12-L.1. Slight coarse bone reaction on left side of D.12-L.1.

FIG. 8. Same case as Fig. 7. Four months later. Erosions have dense borders. Intervertebral bridging of irregular density and outline. Abscess shadow disappeared.

Fig. 9. Same case as Fig. 7. Seven months later. Intervertebral bridging is now broader and denser and its borders are very well defined. Its lower base is wider.

They may be few in number and usually in line, or they may be more numerous and irregularly distributed, thus rendering the structure of the vertebral body into a mottled irregular pattern of translucent and dense areas. In very severe cases, larger and deeper semicircular areas of destruction are present, which open up on the upper or lower surface of the body. Beyond these areas the adjoining parts of the vertebra become cloudy and slightly denser for about one or two centimetres (Fig. 4).

These lesions will take longer to heal and the sclerotic reaction appears later. The destroyed area will be gradually replaced by dense irregular bone. These, however, usually leave a defect in the surface of the body which is very dense at its periphery. Towards the end of the healing phase the adjoining part of the vertebra will become less dense and the the neighbouring vertebra. Such lesions are commonly found in the dorsal region and cause considerable destruction of bone and take a long time to heal (Figs. 5 and 6).

These bear a strong resemblance to Pott's disease and are in many instances accompanied by a paravertebral abscess (Figs. 5 and 7). Out of 20 cases, in which the dorsal spine was involved, there were 14 abscesses noted on radiological examination. There were only two cases with a psoas abscess. Concave erosion of the anterior surface of the body of a vertebra was noted in one case.

\section{(b) Changes in the disc space}

Involvement of the disc with narrowing of the disc space is the general rule. It is an early sign. Only in three cases was the disc space unaltered, yet 


\section{F. Zammit}

the changes in the adjoining vertebrae were quite obvious. In another single case there was narrowing of the disc space with no radiological evidence of bone involvement.

In the early minimallesions it may not be narrowed at first, but eventually its width diminishes after a few weeks. In some cases more than one disc space is involved. The degree of narrowing varies accord-

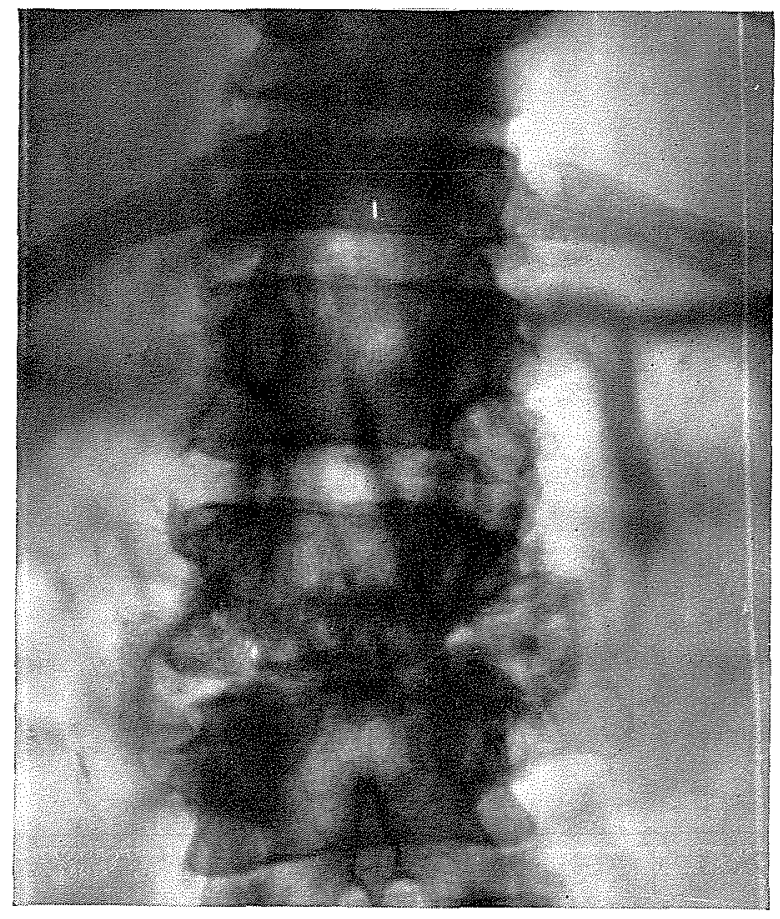

Fiv. 10

S.T, 37 ypars. Signs of cord compression. Laminectomy D.11-12-L.1. Pus obtained from extradural abscess at this site reacted to Bruc. melit. up to $1 / 640$. Culture negative. Animal inoculation did not show presence of mycobact. tuberc. Areas of destruction on both sides of L.2L.3, and at left lower border of L.1 with sclerotic borders. Intervertebral bridging on both sides of L.2-L.3 and on left side of L.1-L.2. Osteophytes on right side of D11-12L.1-2. Collapse of D.12 with normal disc spaces.

ing to the extent of the osseous lesion. Complete narrowing is found when the destructive lesions are numerous and deep and the process has extended to the next vertebra. When the lesion is eccentric the narrowing may not be uniform and there is a scoliosis towards the side of the lesion (Fig. 7).

In several cases the disc spaces above and below the narrowed one, or one of them, were wider than the normal.

Some vertebrae near a narrowed disc space showed slight disalignment of their posterior borders.

Most of the narrowed or obliterated disc spaces regained their width partially or completely during the healing stage and the vertebral list if present was sometimes rectified.

Permanent total disappearance of the disc space with bony ankylosis of the vertebral bodies was not observed. Partial ankylosis of the anterior halves of two vertebral bodies with reappearance of the posterior half of the disc space was noted in one case. This part of the disc remained, however, considerably narrowed.

\section{(c) Proliferative marginal reactions}

These changes are present at different stages of the disease in almost every case of undulant fever spondylitis. They are a very characteristic finding. They are very pronounced as a general rule, but in a small percentage of cases they are not so marked and resemble so much the ordinary osteophytes of osteoarthritis of the spine that they can hardly be distinguished in the chronic cases. Besides, they may be present at other levels away from the main lesion of Brucellosis. In such cases they represent the minor lesion which might have developed at an earlier stage of the disease, especially in relapsing cases (Fig. 4).

The osteophytic outgrowths or intervertebral bridging of undulant fever may have different forms and sizes on either side of an involved vertebra and also at different levels of the spine. They are generally localised to the immediate vicinity of the osteolytic focus.

Their development follows a regular pattern and may be divided into three stages:

(i) At first they start as an ill-defined, faintly opaque, irregular mass of poorly calcified osteoid tissue near the affected border of the vertebra and proceed in the form of a broad arc towards the opposing border of the next vertebra, where similar changes of a lesser degree occur. These two processes may eventually continue to grow and meet or stop growing and remain separated (Figs. 4 and 7).

(ii) In any case their structure will change in a short time into an amorphous heap or band of "woven" bone, which is slightly denser, with irregular margins and of variable shapes (Fig. 8).

They assume the shape of curved beaks when they do not meet, or a continuous bridge of practically uniform width, or a narrow centre and broad bases. The bases in both cases are thicker and wider on the side of the more extensive lesion. When the osseous destruction is of considerable size the base of the bridging next to this lesion is formed of two arms, in $\mathrm{Y}$-shape fashion, enclosing the area of destruction. 


\section{Undulant Fever Spondylitis}

In a few cases a dense oval opacity appeared at the point of convergence of the opposing osteophytic processes, and for some time remained separated from both, but eventually it fused with the advancing heads of these osteophytes and a complete intervertebral bridge was formed (Fig. 2).

Another form of reaction took the shape of a wide triangular spur, like a step, on the anterior surface of the vertebral body, just below a marginal lesion.

In other cases the bridging started at the centre of the border of the vertebra with a sound area between it and the destructive peripheral lesion.

(iii) Eventually the amorphous structure of these proliferative osteophytic processes changes into a lamellar form, becomes denser still and assumes a more orderly trabecular pattern. In several cases the osteophytic processes become narrower and their borders are more clearly defined. The latter transformation takes a longer time to become complete than the previous two phases (Figs. 9 and 10).

As a general rule these proliferative outgrowths take a short time to form. This is a differential point from ordinary osteoarthritis. There were instances where no osteophytes were noticed in the first radiographs of a patient having clinical signs of Brucellar spondylitis, yet in later radiographs they appeared and eventually formed complete intervertebral bridging in two or three months.

They are absent or insignificant in cases of extensive bone destruction. This has been noted more frequently in the dorsal region, where the lesion was severe and accompanied by a paravertebral abscess. Yet even in these cases when the disease approached the healing phase, that is after several months, osteophytic outgrowths appeared and later formed a complete dense regular br dge between the vertebral bodies involved.

Posterior reactions were observed in only two cases. Thickening of the pedicles was noted in one case.

Periosteal thickening at the sides of the body of the vertebra, with apparent widening, occurred as an unusual finding in four cases.

\section{(d) Changes in the intervertebral joints}

Such changes, which have been emphasised by different authors, were very seldom met with in this series. The changes are not convincing in some cases, because all one notices is disappearance of the joint space with hazy outline of the margins. One cannot be sure that such changes are not due to the rotation and list of the vertebrae near the lesion. True erosions were never shown radiologically.

\section{Differential Diagnosis}

The agglutination test was positive in all the cases included in this series: The titre test was done in 33 cases and in 30 of these was found to be high.

The following conditions have to be differentiated from Brucellar spondylitis:

(1) Pott's disease. In several cases it is very difficult to differentiate this disease from Brucellar spondylitis. The tuberculin test and guinea pig inoculation may help. Radiological signs manifest themselves at a later date after the onset. The course of this disease is longer. Bone destruction is more marked and general rarefaction of the diseased bone is more pronounced and more extensive. The healing stage takes a longer time to set in and sclerosis is not a feature. Proliferative bone reactions rarely occur. Gibbus and collapse of the vertebral bodies and later ankylosis are more common. The age incidence is different.

(2) Staphylococcal osteomyelitis. The onset is sudden and the general symptoms are more severe, with high fever and leucocytosis. The interval between the onset and the appearance of radiological signs is shorter, usually about six weeks. There is less new bone formation and sclerosis appears earlier. Bone destruction takes place rapidly and the disc space disappears early. Abscess formation is common. The blood culture and the agglutination test will help to identify the nature of the infection.

(3) Actinomycosis. This produces sclerotic changes with obliteration of the trabecular structure of the vertebrae and long spurs which run longitudinally leading to intervertebral bridging, with little collapse and moderate diminution of the disc space. In this disease, however, discharging sinuses are common and the presence of sulphur granules or the particular organism will render the diagnosis easier.

(4) Scheuermann's disease. In juveniles it is very difficult to distinguish the early case of Brucellar spondylitis from this disease, however, the latter involves more vertebrae and is most common in the lower dorsal region. Schmorl's nodes may be present and there are no proliferative marginal reactions.

(5) Osteoarthritis. The lipping of osteoarthritis is more diffuse and symmetrical. The spurs are well defined, usually smaller, less curved and as a rule they do not meet. Changes in the body of the vertebra next to the osteophytes are rarely seen.

\section{TREATMENT}

Treatment is on conservative lines: in the last few years antibiotics have been used with good results. Most of the patients do well with rest in bed or on a Bradford frame. The pain disappears after a 


\section{F. Zammit}

few weeks. When root pains, with radiation to the upper and lower limbs are present, traction has been applied and the patient is eventually relieved. When the condition of the patient improves and the pain abates or has disappeared a plaster jacket is applied.

Recovery takes place after a period of several months, depending on the extent of the lesion, and in the majority of the cases the patient returns to his former work.

The period of disability varied from three to six months in 60 per cent of cases: in the rest, where the lesion was more extensive, it lasted between six and twelve months. Recovery was not complete before 20 to 24 months in a few cases in which bone destruction was very considerable.

There were only two fatal cases out of this series. One had a cervical lesion with paraplegia; he was admitted to hospital, traction was applied and he died five days later. The other died one month after admission from cerebral haemorrhage.

\section{ACKNOWLEDGMENTS}

I wish to express my thanks to Prof. A. J. Craig and Prof. W. Ganado for their kindness in allowing me access to the records of their cases and also to Sister Alexandra for sorting them out.

\section{SUMMARY}

The general clinical picture of undulant fever is described. The historical and pathological aspects of this disease are briefly reviewed.
The radiological picture of Brucellar spondylitis is based on the review of 62 cases of undulant fever, which showed radiographic evidence of spine involvement.

The main changes are destructive lesions in the vertebral bodies, usually marginal, with characteristic sclerotic reactions around them and proliferative marginal new bone formations resembling calcifications of ligaments or osteophytic processes.

The disc space is usually narrowed. Signs of cord compression were present in eight cases. The differential diagnosis is briefly discussed. Treatment is conservative and in most cases complete cure results.

\section{REFERENCES}

Barcelo, P., Batalla, E., Rotes, J., Vilaseca, J. M., IV Congreso Intern. de Hygiene y Medicina Mediterraneos, 1953.

Bruce, D., Practitioner, 1887, xxix, 161.

Burnett, E., C. R. Acad. Sci., 1922, clxxiv, 421.

Eyre, J. W. H., Lancet, 1908, i, 1747.

Hamilton, P. K., Amer. F. Clin. Path., 1954, xxiv, 580.

Huddleson, I. F., Hardy, A. V., Debono, J. E., Grltner, W., Brucellosis in Man and Animals, 1943 (The Commonwealth Fund, New York).

Kulowski, J., Vinke, T. H., F. Amer. med. Assoc., 1932, 12.

LöfFler, W., Albertini, A., Krankheitsforschung, 1930, viii, 1 .

Meyer, K. F., Essays in Biology, 1943, 437 (University of California Press).

Rabson, S. M., Amer. F. Clin. Path., 1939, ix, 604.

Rainsford, S. G., F. R. nav. med. Serv., 1935, xxi, 81.

Sharp, W. B., Arch. Path., 1934, 1xxii, 108.

Sundberg, R. D., and Spink, W. W., Blood Spec., $1947, \mathrm{i}, 7$.

Wegener, F., Allg. Pathol., 1935, lxiv, 33.

Wohlwill, F., Virchows Arch., 1932, cclxxxvi, 141. 1214.

Wright, A. F., and Semple, D., Brit. med. F., 1897, i,

ZAMMIT, T., Reports of the Commission on Mediterranean Fever, 1905, 83 . 